\title{
IMPROVED LOSSLESS CODING ALGORITHM IN H.264/AVC BASED ON HIERARCHICAL INTRA PREDICTION
}

\author{
Li-Li Wang and Wan-Chi Siu \\ Department of Electronic and Information Engineering \\ The Hong Kong Polytechnic University \\ Hung Hom, Kowloon, Hong Kong
}

\begin{abstract}
In this paper, an improved lossless intra prediction algorithm based on H.264/AVC framework is proposed. In the new algorithm, samples in a macroblock/block are hierarchically predicted, instead of using a block-based prediction as a whole. More specifically, four groups are extracted from the samples in the MB/block. Samples in the first group are firstly predicted based on directional intra prediction method, and then samples in other groups are predicted using the samples in the first group as the reference. As a result, the information left in the residual block can be reduced since the samples can be predicted accurately by using nearer references. Experimental results show that compared with other methods in the literature the proposed algorithm gives a much better compression performance with the lowest encoding complexity.
\end{abstract}

Index Terms - Lossless video coding, H.264/AVC, hierarchical intra prediction, intra coding

\section{INTRODUCTION}

Lossless coding technique plays an important role in perfectly preserving valuable information using less storage space. This technique was initially developed for image coding and standardized as JPEG lossless coding [1]. It has many applications, such as medical images, digital archives, digital documentations and so on. With the development of video coding standards, the lossless coding technique is also integrated into video coding standards, such as the H.264/AVC standard [2,3]. The lossless coding method used in H.264/AVC standard can be considered as the state-of-art representative which is applied to the video coding standard. Since there is no transform and quantization in lossless video coding, the encoding complexity can be reduced substantially but more bits are needed to encode video sequences. Another problem is that, since the H.264/AVC has a block-based coding structure [4], samples away from the references cannot be predicted well due to poor correlation. Therefore, further improvement of lossless coding method in H.264/AVC standard is always desirable for the purpose of compression.
Recently, researchers have made various studies on the improvement of lossless compression algorithm in the H.264/AVC standard. Lee et al.[5] proposed a lossless intra prediction method based on the samplewise DPCM instead of using a block-based approach. This algorithm achieves better performance since near samples are used as the reference. However, the samplewise DPCM method can only be applied to the modes with one sample predictor. As a result, the performance is thus limited. Seeing this problem, in [6], each pixel in the current $\mathrm{MB} /$ block is predicted by the immediate samples adjacent to the current sample using a linear interpolation concept. As a result, the samplewise DPCM concept can be easily extended to other prediction modes. Experimental results implemented by us show that the lossless compression performance can be further improved compared with the algorithm in [5]. Note that the above two methods almost have the same encoding complexity compared with that in H.264/AVC standard.

In this paper, a hierarchical intra prediction (HIP) method is suggested to improve the lossless compression efficiency and also to reduce the encoding complexity of an encoder. We classify samples in a macroblock into four groups. The samples in the first group are firstly predicted using the directional intra prediction method based on samplewise DPCM, and then the left samples in other groups can be predicted adaptively based on the minimal gradient between neighboring references. Experimental results show that, this method can solve the inaccuracy of the intra prediction, and thereby the coding efficiency can be enhanced when compared with other lossless intra coding methods [5,6]. Furthermore, since only part of samples are predicted using the rate optimization (RO) method, the computational complexity can be reduced significantly as compared with other methods $[5,6]$.

\section{LOSSLESS INTRA CODING IN H.264/AVC}

In FRExt high profiles of H.264/AVC standard [3], a transform-bypass lossless coding method was proposed to improve the efficiency of the lossless coding. This method uses intra prediction and entropy coding for encoding sample values. Different from the lossy video coding in H.264/AVC, the residual block is directly scanned and encoded by entropy coding without transformation or 
quantization in the process of lossless coding. However, the intra prediction algorithm used to produce the residual block is the same for both lossless coding and lossy coding. In FRExt high profiles, four basic types of intra prediction for luma component are available: 16x16 luma prediction (I16MB type), $8 \times 8$ luma prediction (I8MB type) and $4 \times 4$ luma prediction (I4MB type) and IPCM[2,3]. There are four prediction modes in the I16MB type and 9 prediction modes in the I4MB/I8MB types, which are shown in figs. 1 and 2. Chroma component can be predicted using four modes, which are similar to those in I16MB type.

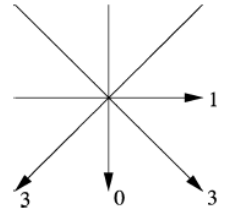

(a)

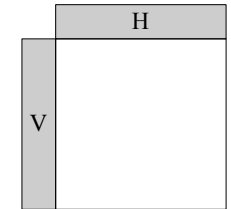

(b)
Fig.1 (a) Three mathematical prediction directions for I16MB type,

(b) Reference pixels for I16MB type

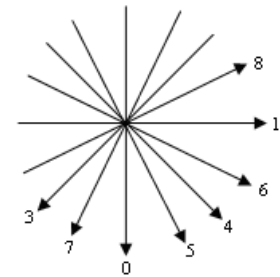

(a)

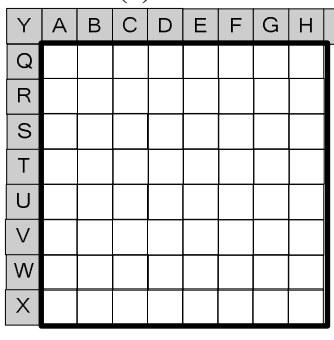

(c)

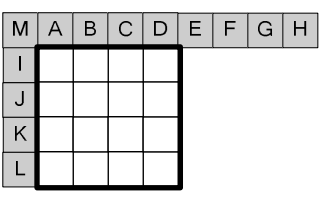

(b)

\section{\begin{tabular}{l|l|l|l|l|l|l|} 
J & K & L & M & N & O & P \\
\hline
\end{tabular}}

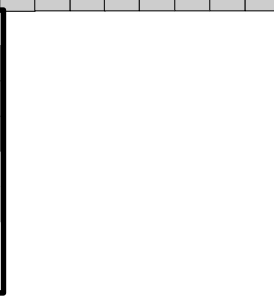

Fig.2 (a) Eight prediction directions for I4MB/I8MB type, (b) Reference pixels from A-M for I4MB type, (c) Reference pixels from A-Y for I8MB type

The new lossless coding method makes the encoder very efficient. However, we also note that the intra block/MB is predicted as a whole by an extrapolation of the neighboring reconstructed pixels in H.264/AVC standard. As a result, the samples which are far from their references may not be predicted well due to poor correlation. Especially for the blocks predicted using the I8MB or I16MB type, the poor prediction is more obvious since a larger block size is used.

\section{PROPOSED ALGORITHM BASED ON HIERARCHICAL INTRA PREDICTION}

With a notice of the problem mentioned above, we propose in this paper a hierarchical intra prediction (HIP) method for I8MB type, I16MB type and Chroma prediction in this section. Details are shown as follows.

\subsection{Improved HIP method for I8MB type}

In FRExt profiles, I8MB type is introduced to improve the encoding efficiency of the intra prediction. A MB is firstly divided into four $8 \times 8$ blocks. For each $8 \times 8$ block, nine prediction modes are used to do intra prediction. In order to improve the prediction accuracy for samples away from their references, we suggest that the pixels in each $8 \times 8$ block be predicted hierarchically instead of using the whole-blockbased method in the original H.264/AVC standard. Four groups from $\mathrm{G} 0$ to $\mathrm{G} 3$ in each $8 \times 8$ block are classified as shown in fig.3. By sampling with the corresponding group numbers as shown in fig. 3 (a), four groups are formed as shown in fig.3 (b) for the residual entropy coding process.

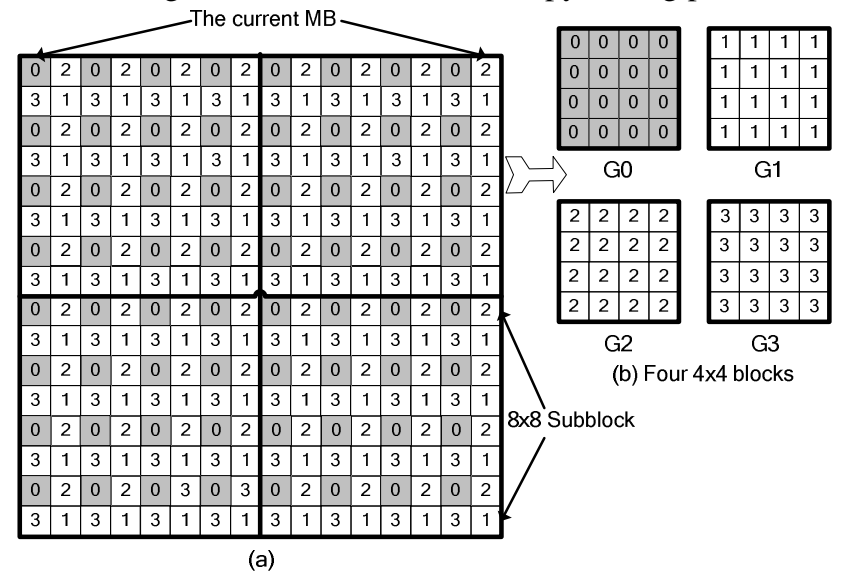

Fig.3 Structure of HIP for I8MB type

The detailed prediction strategies of samples in the four groups are presented as follows:

First, intra prediction for the samples in G0 using nine prediction modes of I8MB type is made.

According to the nine prediction modes of the I8MB type in H.264/AVC, the predicted values of the 16 samples labeled as 0 in fig. 3 are computed using the samplewise DPCM based method [5] instead of block based method in original H.264/AVC. After the samples in G0 are predicted, the residual information is scanned in a $4 \times 4$ zig-zag mode instead of the $8 \times 8$ mode. And then the rate (bits) used to represent the scanning coefficients and the best prediction mode are obtained by entropy coding. The mode with the minimal rate is selected as the prediction mode of G0. For a lossless coding process, there is no degradation in PSNR, the samples can be reconstructed without any loss of fidelity. As a result, the mode with the minimal rate can make the best compression efficiency for an encoder.

Second, intra prediction for the samples in G1,G2 and G3 based on the measure of intensity gradient is then made

The remaining 48 pixels in the $8 \times 8$ block are classified into three groups (G1, G2 and G3), which correspond to three $4 \times 4$ blocks as shown in fig. 3 (b). Samples in G1 are firstly predicted and reconstructed using samples in G0 as references. Subsequently, samples in G2 and G3 are predicted and reconstructed using samples in G0 and G1. 
The predicted values of samples in these three $4 \times 4$ blocks are obtained adaptively based on a measure of the gradients along different directions. The arrangement is illustrated using (1)-(3) in fig.4.

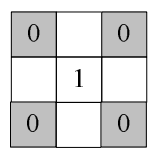

(a)

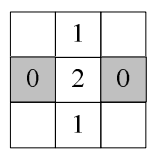

(b)

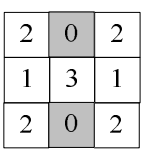

(c)
Fig.4 Predicted structures of samples in group 1,2, and 3 (a)group 1 (b)group 2 (c)group 3

We then have the following formulations.

$$
\begin{aligned}
& p_{i, j}^{1}=\left\{\begin{array}{cc}
\left(f_{i-1, j-1}^{0}+f_{i+1, j+1}^{0}+1\right)>>1, & \left|f_{i-1, j-1}^{0}-f_{i+1, j+1}^{0}\right| \\
\left(f_{i+1, j-1}^{0}+f_{i-1, j+1}^{0}+1\right)>>1, & <\left|f_{i+1, j-1}^{0}-f_{i-1, j+1}^{0}\right|
\end{array} \mid\right. \\
& p_{i, j}^{2}=\left\{\begin{array}{lc}
\left(f_{i-1, j}^{0}+f_{i+1, j}^{0}+1\right)>>1, & \text { otherwise } \\
\left(f_{i-1, j}^{0}-f_{i+1, j}^{0}|<| f_{i, j-1}^{1}-f_{i, j+1}^{1} \mid\right.
\end{array}\right.
\end{aligned}
$$

$$
p_{i, j}^{3}=\left\{\begin{array}{cc}
\left(f_{i-1, j}^{1}+f_{i+1, j}^{1}+1\right)>>1, & \left|f_{i-1, j}^{1}-f_{i+1, j}^{1}\right|<\left|f_{i, j-1}^{0}-f_{i, j+1}^{0}\right| \\
\left(f_{i, j-1}^{0}+f_{i, j+1}^{0}+1\right)>>1, & \text { otherwise }
\end{array}\right.
$$

where $p_{i, j}^{m}$ denotes the predicted value of the sample in the position (i,j) of group $\mathrm{m}(\mathrm{Gm})$, and $f_{i, j}^{m}$ denotes the reconstructed value of the reference sample in the position (i,j) of $\mathrm{Gm}$.

Since the best predicted direction (horizontal or vertical/diagonal down left or diagonal down right) can be determined adaptively based on the measure of intensity gradient of the neighboring reconstructed samples, there is no extra bit needed to encode the predicted direction. Another observation is that the samples in the last three groups can adaptively select the best prediction directions according to the minimal gradients. As a result, the prediction directions of the samples in a block may be different using the proposed algorithm. This cannot be obtained using previous intra prediction methods[5-7]. Furthermore, since samples in G1, G2 and G3 are predicted using the neighboring reconstructed samples which are equal to the original samples for lossless coding, the correlation between them is generally higher. As a result, the information left in the residual block is reduced, and only a smaller number of bits are needed to encode the residual block.

\subsection{Improved HIP method for I16MB type /Chroma prediction}

Similar to I8MB type (except now the block size is $16 \times 16$ ), the whole macroblock with a $16 \times 16$ size is classified into four groups from G0 to G3. Samples in G0 are predicted using four modes in I16MB type based on samplewise DPCM method [5]. The mode with the minimal rate is selected and encoded. Samples in G1,G2 and G3 are then predicted using the neighboring reconstructed samples as (1)-(3). The prediction of Chroma cr/cb block with an $8 \times 8$ size can also be performed in a similar way to the HIP method of the I16MB type.

\subsection{Further improvement of HIP method}

Compared with the I8MB type and I16MB type, the I4MB type is associated with a $4 \times 4$ block, which is small. Experimental results show that the HIP method cannot have a better effect on I4MB type. Therefore, the method similar to [6] is used to improve the prediction of I4MB type for lossless coding. The only difference between them is the prediction of DC mode. According to some simulation work, we propose the following idea to improve the prediction of DC mode in every intra prediction type (including I4MB, I8MB, I16MB and Chroma prediction) according to the availability of the neighboring reference pixels.

$$
p_{i, j}^{0}=\left\{\begin{array}{cc}
\left(f_{i, j-1}^{0}+f_{i-1, j}^{0}+1\right)>>1, & \text { up_available } \\
\left(f_{i-1, j}^{0}+f_{i-1, j+1}^{0}+1\right)>>1, & \text { left_available } \\
128, & \text { otherwise }
\end{array}\right.
$$

where coordinates (i,j) correspond to samples in G0. Similarly, in order to use the concept of DPCM for the plane mode in the I16MB type and the Chroma prediction, the predicted value of the sample in G0 for plane mode is given as follows.

$$
p_{i, j}^{0}=\left(f_{i, j-1}^{0}+f_{i+1, j}^{0}+1\right)>>1
$$

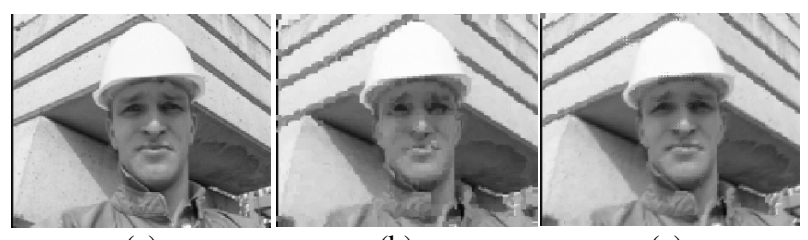

(a)

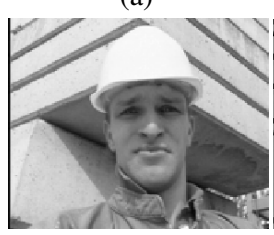

(d) (b)

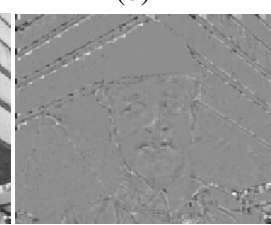

(e) (c)

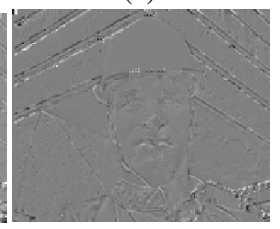

(f)
Fig.5 Experimental results on Luma component for the first frame of Foreman.QCIF sequence (a) Original frame, (b) Predicted frame based on JM reference software of H.264/AVC standard, (c) Predicted frame based HIP, (d) Reconstructed frame, (e) Residual frame based on JM, and (f) Residual frame based HIP

Fig. 5 shows an example of original frame, reconstructed frame, predicted frame and their corresponding residual frames for two methods. From the results of the predicted frames, it is clear that the method based on HIP makes a better prediction than that of the block-based method in 
H.264/AVC standard, and thereby the information left in the residual frame is reduced.

\section{EXPERIMENTAL RESULTS}

In order to evaluate the performance of the proposed algorithm, reference software JM 12.2[7] was used to carry out experiments on several YUV 4:2:0 format sequences. A total of 150 frames were encoded for each sequence. The test was based on the FRExt high profiles with specifications provided in [8]. All frames are intra coded. The frame rate was set to 30 . CAVLC entropy coding method was used for the experiments. Deblocking was not used for lossless coding.

Since there is no degradation in video quality for lossless coding, we only present the compression ratios for different lossless intra prediction methods in terms of encoding bitrates for sequences of different sizes in tables III-V. It can be observed from these tables, the method based on samplewise DPCM makes a great compression ratio than the block-based method in the original H.264/AVC standard. With the improvements of more intra coding modes, the method based on SI_DPCM achieves a further compression compared with samplewise DPCM method. However, the encoding complexities of the above two methods are almost the same as H.264/AVC. Compare with the two methods, the proposed method can achieve a consistent increase in compression ratios for most of the test sequences. Note that only part of samples are predicted using rate optimization ( $\mathrm{RO}$ ) process based on the proposed algorithm. Hence the complexity of the encoder can be reduced using the proposed algorithm compared with the method in H.264/AVC standard.

Table III Results of compression ratios for QCIF sequences

\begin{tabular}{|c|c|c|c|c|}
\hline \multirow{2}{*}{$\begin{array}{c}\text { Sequence } \\
\text { (QCIF) }\end{array}$} & $\begin{array}{c}\text { Bitrate } \\
\text { (kbits/s) }\end{array}$ & \multicolumn{3}{|c|}{$\Delta$ Bitrate [\%] } \\
\cline { 2 - 5 } & H.264_LS & $\begin{array}{c}\text { H.264 } \\
\text { DPCM }\end{array}$ & $\begin{array}{c}\text { SI_- } \\
\text { DPCM }\end{array}$ & $\begin{array}{c}\text { Our } \\
\text { method }\end{array}$ \\
\hline \hline Coastguard & 5855.984 & 10.574 & 11.846 & 12.313 \\
\hline Monitor & 5377.597 & 11.853 & 14.863 & 15.588 \\
\hline Foreman & 5566.776 & 9.026 & 11.123 & 11.628 \\
\hline Akiyo & 4659.207 & 10.843 & 15.904 & 17.365 \\
\hline GrandMother & 5306.482 & 9.132 & 11.572 & 12.934 \\
\hline \hline Average & $\mathbf{5 3 5 3 . 2 0 9}$ & $\mathbf{1 0 . 2 8 6}$ & $\mathbf{1 3 . 0 6 2}$ & $\mathbf{1 3 . 9 6 6}$ \\
\hline
\end{tabular}

Table IV Results of compression ratios for CIF sequences

\begin{tabular}{|c|c|c|c|c|}
\hline \multirow{2}{*}{$\begin{array}{c}\text { Sequence } \\
\text { (CIF) }\end{array}$} & $\begin{array}{c}\text { Bitrate } \\
\text { (kbits/s) }\end{array}$ & \multicolumn{3}{|c|}{$\Delta$ Bitrate [\%] } \\
\cline { 3 - 6 } & H.264_LS & $\begin{array}{l}\text { H.264 } \\
\text { DPCM }\end{array}$ & $\begin{array}{c}\text { SI_ } \\
\text { DPCM }\end{array}$ & $\begin{array}{c}\text { Our } \\
\text { method }\end{array}$ \\
\hline \hline Foreman & 19353.068 & 8.083 & 10.410 & 11.363 \\
\hline Container & 20372.872 & 8.152 & 10.711 & 11.268 \\
\hline Silent & 21750.124 & 8.450 & 12.036 & 12.532 \\
\hline Tempete & 26259.104 & 9.532 & 13.135 & 13.890 \\
\hline Waterfall & 27073.632 & 7.946 & 12.964 & 14.083 \\
\hline \hline Average & $\mathbf{2 2 9 6 1 . 7 6 0}$ & $\mathbf{8 . 4 3 3}$ & $\mathbf{1 1 . 8 5 1}$ & $\mathbf{1 2 . 6 2 7}$ \\
\hline
\end{tabular}

Table V Results of compression ratios for HDTV sequences

\begin{tabular}{|c|c|c|c|c|}
\hline \multirow{2}{*}{$\begin{array}{c}\text { Sequence } \\
\text { (HDTV) }\end{array}$} & $\begin{array}{c}\text { Bitrate } \\
\text { (kbits/s) }\end{array}$ & \multicolumn{3}{|c|}{$\Delta$ Bitrate [\%] } \\
\cline { 2 - 5 } & H.264_LS & $\begin{array}{c}\text { H.264 } \\
\text { DPCM }\end{array}$ & $\begin{array}{c}\text { SI__ } \\
\text { DPCM }\end{array}$ & $\begin{array}{c}\text { Our } \\
\text { method }\end{array}$ \\
\hline \hline Sunflower & 168292.240 & 15.361 & 22.372 & 22.366 \\
\hline Crowdrun & 241153.440 & 5.462 & 8.035 & 9.517 \\
\hline InToTree & 198065.424 & 2.210 & 2.809 & 4.983 \\
\hline OldTownCross & 199703.840 & 4.528 & 4.661 & 6.503 \\
\hline \hline Average & $\mathbf{2 0 1 8 0 3 . 7 0 0}$ & $\mathbf{6 . 8 9 0}$ & $\mathbf{9 . 4 6 9}$ & $\mathbf{1 0 . 8 4 2}$ \\
\hline
\end{tabular}

\section{CONCLUSION}

In this paper, an algorithm based on a hierarchical intra prediction for lossless coding is presented. Based on the hierarchical prediction, some pixels in an $\mathrm{MB} / \mathrm{block}$ are firstly predicted and encoded with a minimal rate, and the others are then predicted adaptively based on a minimal gradient approach using the nearer references. Experimental results show that the proposed algorithm can improve the coding efficiency of the lossless coding in the H.264/AVC standard. It is certainly better than other algorithms[5-7]. In fact, there is still some room of improving the efficiency of this lossless coding method. For example, having a better prediction method for samples in G1, G2 and G3 can result in less information in the residual block. Furthermore, a better entropy coding strategy is equally important for the resultant residual block to be compressed efficiently.

Acknowledgment: This work is supported by the Centre for Multimedia Signal Processing (1-BB9D), Hong Kong Polytechnic University and the Research Grant Council of the Hong Kong SAR Government (PolyU 5267/07E).

\section{REFERENCES}

[1]W.B. Pennebaker and J.L. Mitchell, JPEG: Still Image Data Compression Standard, Van Nostrand Reinhold, New York, 1992.

[2]ITU-T Rec. H.264/ISO/IEC 11496-10, Advanced video coding, Final Committee Draft, Document JVTG050, Mar.2003.

[3]G.J. Sullivan, P. Topiwala, and A. Luthra (2007), The H.264/AVC Advanced Video Coding Standard: Overview and Introduction to the Fidelity Range Extensions. Retrieved Oct. 2009.

[4]L.L.Wang and W.C.Siu, " H.264 fast intra mode selection algorithm based on direction difference measure in the pixel domain ," ICASSP, pp.1037-1040, Apr. 2009.

[5]Y.L. Lee, K.H. Han and G.J. Sullivan, "Improved lossless intra coding for H.264/MPEG-4 AVC," IEEE Trans. Image Process., vol.15, pp.2610-2615, Sept. 2006.

[6]S.T. Wei, S.R. Shen, B.D. Liu and J.F. Yang, "Lossless image and video coding based on H.264/AVC intra predictions with simplified interpolations," ICIP, pp.633-636, Nov. 2009.

[7]JVT H.264/AVC Reference Software version JM 12.2, http://iphome.hhi.de/suehring/tml/download/old_jm/

[8]G. Sullivan, "Recommended Simulation Common Conditions for Coding Efficiency Experiments Revision 1", 31st VCEGAE010R1 meeting, Marrakech, Morocco, 13-19 January, 2007. 